and secondary dermatomiositis) (DM) and polymyositis (PM) including the rest of the patients. Also, IIM were classified (II) as primary polimiositis (PPM), primary dermatomyositis (PDM), overlap syndrome (OSd), juvenile myopathies (JM), cancer-associated myopathies (CAM), autoimmune necrotizing myopathy and inclusion body myositis (these were grouped as other myositis; OM).

Results: In the PM and DM groups 250 and $229(52.2 \%$ and $47.8 \%)$ patients were included respectively. Positive ANA, anti-Jo1 and anti-RNP were higher in the PM than in the DM group $(67,22$ and $19 \%$ vs. 56,11 and $6 \%)(p=0.21$, $\mathrm{p}=0.002, p=0.0001$, respectively). The presence of anti-MI2 was higher in the DM group ( $\mathrm{p}=0.024)$, according to the classification II, we found statistically significant differences in ANA, anti-Jo1, anti-RNP and anti-ACA. The OSd group had the highest proportion of ANA, anti-RNP and ACA positive AA and the JM group had the lowest frequency of Anti-Jo1 (see Table 1).

Table 1. Autoantibodies profile in IIM according to classification II

\begin{tabular}{|c|c|c|c|c|}
\hline AA & Compariso & n groups & Frequency & $\mathbf{P}$ \\
\hline \multirow{5}{*}{ ANA } & \multirow{4}{*}{ OSd vs. } & PPM & $91 \%$ vs. $54 \%$ & \multirow{5}{*}{0,0001} \\
\hline & & PDM & $91 \%$ vs. $63 \%$ & \\
\hline & & $\mathrm{JM}$ & $91 \%$ vs. $40 \%$ & \\
\hline & & CAM & $91 \%$ vs. $63 \%$ & \\
\hline & PDM vs. & $J M$ & $63 \%$ vs. $54 \%$ & \\
\hline \multirow{3}{*}{ Anti-Jo1 } & \multirow{3}{*}{ JM vs. } & PPM & $3 \%$ vs. $25 \%$ & \multirow{3}{*}{0,0001} \\
\hline & & PDM & $3 \%$ vs. $19 \%$ & \\
\hline & & $\mathrm{OM}$ & $3 \%$ vs. $36 \%$ & \\
\hline \multirow{4}{*}{ Anti-RNP } & \multirow{4}{*}{ OSd vs. } & PPM & $40 \%$ vs. $6 \%$ & \multirow{4}{*}{0,0001} \\
\hline & & PDM & $40 \%$ vs. $3 \%$ & \\
\hline & & $\mathrm{JM}$ & $40 \%$ vs. $7 \%$ & \\
\hline & & CAM & $40 \%$ vs. $5 \%$ & \\
\hline ACA & OSd vs. & PDM & $18 \%$ vs. $3 \%$ & 0,002 \\
\hline
\end{tabular}

Conclusions: The AA associated with the IIM subtypes is consistent with published scientific evidence on other cohorts for ANA, anti-Jo1, anti-RNP and anti-MI2, in spite of the small sample size. The OSd group showed higher ANA and anti-RNP frequencies which might be explained by the coexistence of SLE and MCTD patients. It could be interesting to follow up those PPM patients with positive AA because they could be in the future diagnosed with a connective tissue disease. Carrying out longitudinal studies that include a greater proportion of patients may help to evaluate and predict the clinical course of IIM.

References:

[1] Bohan A, Peter JB. N Engl J Med 1975 Feb 13;292(7):344-7.

Acknowledgements:.

Disclosure of Interest: None declared

DOI: 10.1136/annrheumdis-2017-eular.7050

\section{AB0626 EPITOPE PROFILING OF ANTI-RO52 ANTIBODIES IN PATIENTS WITH SYSTEMIC SCLEROSIS, SYSTEMIC SCLEROSIS-ASSOCIATED PRIMARY BILIARY CIRRHOSIS, AND PRIMARY BILIARY CIRRHOSIS ALONE}

C. Liaskos ${ }^{1}$, A. Gkoutzourelas ${ }^{1}$, T. Simopoulou ${ }^{1}$, E. Marou ${ }^{1}$, T. Scheper ${ }^{2}$, W. Meyer ${ }^{2}$, L. Sakkas ${ }^{1}$, D.P. Bogdanos ${ }^{1} .{ }^{1}$ Rheumatology and Clinical Immunology, University of Thessaly, School of Health Sciences, Larisa, Thessaly, Greece; ${ }^{2}$ Institute of Immunology, Euroimmun AG, Lubeck, Germany

Background: Anti-Ro52 antibodies are detected in patients with Sjogren's syndrome (SjS), systemic sclerosis (SSc) and other autoimmune rheumatic diseases. Epitope mapping studies of anti-Ro52 in autoimmune rheumatic diseases have failed to find any difference on epitope recognition suggesting a common stimulus for the loss of tolerance to Ro52. Such epitope differences, however, were noted between SjS and non-rheumatic diseases and in particular, SjS-associated primary biliary cirrhosis (SjS-PBC) or PBC alone (1).

Objectives: To assess whether or not the Ro52 epitope profile in anti-Ro52+ SSc patients differs from that of SSc-associated PBC (SSc-PBC) or PBC.

Methods: Serum samples were obtained from 63 anti-Ro52-positive (by ELISA) patients (33 SSc, 10 SSc-PBC, 20 PBC alone). Antibody reactivity to full length recombinant Ro52 and 5 baculovirus expressed Ro52 fragments spanning the whole sequence [Ro52-1 (aa 1-129), Ro52-2 (aa 125-268), Ro52-3 (aa 269475), Ro52-4 (aa 57-180, partly overlapping with Ro52-1 and Ro52-2), and Ro52-5 (aa 181-320, partly overlapping with Ro52-2 and Ro52-3)] were tested by a line immunoassay.

Results: Reactivity was present to: full length Ro52 in all anti-Ro52 positive SSc, PBC-SSc, or PBC patients by line immunoassay; Ro52-1 in 6/33 (18.2\%) SSc, $3 / 10(30 \%)$ SSc-PBC, and 3/20 (15\%) PBC; Ro52-2 in 33/33 (100\%) SSc, $10 / 10(100 \%)$ SSc-PBC and 20/20 (100\%) PBC; Ro52-3 in 0/33 (0\%) SSc, 0/10 (0\%) SSc-PBC and 0/20 (0\%) PBC; Ro52-4 in 4/33 (12.1\%) SSc, 2/10 (20\%) SSc-PBC, and $4 / 20(20 \%)$ PBC and Ro52-5 in $11 / 33$ (33.3\%) SSc $4 / 10(40 \%)$ SSc-PBC, and $9 / 20$ (45\%) PBC respectively ( $p>0.05$ for all epitopes amongst the 3 cohorts). No statistically significant correlation was found.

Conclusions: The major epitope of anti-Ro52 does not differ among SSc, PBC-SSc and PBC and is localized on aa125-268, the major epitopic region of anti-Ro52 antibodies in SjS and other rheumatic diseases. Our epitope mapping data suggest that the mechanisms responsible for the loss of tolerance to Ro52 is common amongst diseases.

\section{References:}

[1] Mytilinaiou, Maria G.; Meyer Wolfgang; Komorowski, Lars; Probst, Christian; Davies, Edward T.; Vergani, Diego; Bogdanos, Dimitrios P. B-cell epitope mapping of anti-Ro52 responses in patients with primary biliary cirrhosis., Hepatology, Vol. 52 (4), 2010, 489.

Disclosure of Interest: C. Liaskos: None declared, A. Gkoutzourelas: None declared, T. Simopoulou: None declared, E. Marou: None declared, T. Scheper Employee of: Euroimmun AG, W. Meyer Employee of: Euroimmun AG, L. Sakkas: None declared, D. Bogdanos: None declared

DOI: 10.1136/annrheumdis-2017-eular.5299

\section{AB0627 RISE-SSC: A DOUBLE-BLIND, RANDOMISED STUDY EVALUATING THE EFFICACY AND SAFETY OF RIOCIGUAT FOR THE TREATMENT OF PATIENTS WITH DIFFUSE CUTANEOUS SYSTEMIC SCLEROSIS}

D. Khanna ${ }^{1}$, Y. Allanore ${ }^{2}$, C. Denton ${ }^{3}$, M. Matucci-Cerinic ${ }^{4}$, J. Pope $^{5}$, Z. Yao ${ }^{6}$, J. Curram ${ }^{7}$, J. de Oliveira Pena ${ }^{8}$, O. Distler ${ }^{9} .{ }^{1}$ University of Michigan, Ann Arbor, United States; ${ }^{2}$ Paris Descartes University, Paris, France; ${ }^{3}$ University College London, London, United Kingdom; ${ }^{4}$ University of Florence, Florence, Italy; ${ }^{5}$ University of Western Ontario, Ontario, Canada; ${ }^{6}$ Bayer AG, Beijing, China; ${ }^{7}$ Bayer plc, Newbury, United Kingdom; ${ }^{8}$ Bayer AG, Whippany, New Jersey, United States; ${ }^{9}$ University Hospital of Zürich, Zürich, Switzerland

Background: Diffuse cutaneous systemic sclerosis (dcSSc) is a severe subtype of SSc, a rare, chronic autoimmune connective tissue disease associated with significant morbidity and mortality, and with reduced quality of life. Currently, there are no approved disease-modifying therapies to treat the vascular and fibrotic damage in patients with dcSSc. Soluble guanylate cyclase stimulators, such as riociguat, target the transforming growth factor beta pathway, inhibiting signalling through non-SMAD pathways. They have been shown to inhibit dermal fibrosis in several animal models and to ameliorate fibrosis of the skin and intestine in bleomycin-induced fibrosis and experimental chronic graft-versushost disease. ${ }^{1}$ These preclinical data suggest that riociguat has vascular and antifibrotic properties that may be beneficial in dcSSc, potentially delaying disease progression.

Objectives: RISE-SSc (NCT02283762) is an international, multicentre, randomised, double-blind, placebo-controlled, parallel-group, Phase II study to assess the efficacy and safety of treatment with riociguat versus placebo in patients with dcSSc. The primary efficacy endpoint is change in modified Rodnan skin score (mRSS) from baseline to Week 52.

Methods: Eligibility criteria include a disease duration of $\leq 18$ months and mRSS of $10-22$, parameters identified to be predictors of progression of skin fibrosis. ${ }^{2}$ Patients were randomised (1:1) to treatment with riociguat (adjusted up to $2.5 \mathrm{mg}$ tid over the first 10 weeks) or placebo for a total of 52 weeks. The double-blind phase will be followed by an open-label extension phase for up to 6 years where all patients receive riociguat.

Results: Study recruitment was completed on $30 \mathrm{Dec} 2016$ and 121 patients have been randomised. Mean \pm SD age at inclusion was $50.7 \pm 12.3$ years (range $18-79$ ) and $76 \%(n=92)$ were female. At baseline the mean \pm SD mRSS was $16.8 \pm 3.7$ (range 10-22) and disease duration was $8.8 \pm 5.5$ months (range 0.5-18.5). Almost $70 \%$ of the patients tested positive for either anti-SCL-70 (43\%) or anti-RNA polymerase III (25\%) antibodies, while $32 \%$ of patients tested negative for these two antibodies. Swollen and tender joint counts were present in $38(31.4 \%)$ and $51(42.1 \%)$ patients, respectively, while 35 patients $(28.9 \%)$ had tendon friction rubs. Digital ulcers were present in 15 patients $(12.4 \%)$ at baseline.

Conclusions: RISE-SSc has a unique trial design with a cohort enriched for disease progressors, ${ }^{2}$ which is reflected in the baseline characteristics of the randomised patients. Results of the RISE-SSc study will provide data to inform not only the efficacy, safety and tolerability of riociguat treatment in patients with dcSSc, but also the feasibility of a new trial design based on cohort enrichment. References:

[1] Khanna D, et al. J Scleroderma Relat Disord 2016;1:186-93.

[2] Maurer B et al. Ann Rheum Dis 2015;74:1124-31.

Disclosure of Interest: D. Khanna Grant/research support from: Bayer, BMS, Genentech/Roche, Sanofi-Aventis, NIH K24AR063120, Consultant for: Actelion, Bayer, Covis, Cytori, EMD Serono, Genentech/Roche, Gilead, GSK, SanofiAventis, Y. Allanore Grant/research support from: BMS, Genentech-Roche, Inventiva, Pfizer, Sanofi, Consultant for: Actelion, Bayer, Biogen, GenentechRoche, Galapagos, Medac, Pfizer, Sanofi, Servier, UCB, C. Denton Grant/research support from: CSL Behring, Bayer, GSK, Inventiva, Consultant for: Actelion, Bayer, GSK, Merck-Serono, Genentech-Roche, Inventiva, Sanofi-Aventis, Boehringer Ingelheim, M. Matucci-Cerinic Grant/research support from: BMS, Pfizer, Actelion, MSD, Lilly, Speakers bureau: Pfizer, BMS, Actelion, Lilly, J. Pope Grant/research support from: Bayer, Merck, Consultant for: BMS, Roche, Bayer, Merck, Z. Yao Employee of: Bayer Healthcare Company Ltd, Beijing, China, J. Curram Shareholder of: Bayer AG, Employee of: Bayer plc, J. de Oliveira Pena Employee of: Bayer US LLC, O. Distler Grant/research support from: Actelion, Bayer, Boehringer Ingelheim, Pfizer, Sanofi, Consultant for: 4 D Science, Actelion, Active Biotec, Bayer, Biogenldec, BMS, Boehringer Ingelheim, ChemomAb, EpiPharm, espeRare foundation, Genentech/Roche, GSK, Inventiva, Lilly, medac, Mepha, Medlmmune, Mitsubishi Tanabe Pharma, Pharmacyclics, Pfizer, Sanofi, Serodapharm, Sinoxa, Speakers bureau: AbbVie, iQone Healthcare, Mepha 
DOI: 10.1136/annrheumdis-2017-eular.3718

\section{AB0628 MACROVASCULAR DYSFUNCTION OF UPPER AND LOWER LIMBS CORRELATES WITH DIGITAL ULCERATIONS IN PATIENTS WITH SYSTEMIC SCLEROSIS}

E. Moradkhan ${ }^{1}$, S. AleSaeidi ${ }^{2}$, F. Gharibdoost ${ }^{3} .{ }^{1}$ Shahid Beheshti University of Medical Siences; ${ }^{2}$ Rheumatology, Internal medicine, Tehran university of medical sciences, Medical Faculty; ${ }^{3}$ Tehran university of medical siences, Tehran, Iran, Islamic Republic Of

Background: Systemic sclerosis (SSc) is a chronic connective tissue disorder of unknown etiology characterized by tissue fibrosis and vascular damage [1]. Digital ulcerations (DUs) are common manifestations of vascular involvement [2]. Althoughthe vascular dysfunction of SSc has been considered mainly to affect microvasculature [3], there is recent evidence showing that SSc is associated with the prevalence of large vessel disease [4]. However, only a few studies investigated the relationship between macrovascular disease and its role in the clinical manifestations of SSc such as ulcers.

Objectives: To assess the relationship between the Macrovascular dysfunction of upper and lower limbs with digital ulcerations in patients with systemic sclerosis. Methods: Ninety patients with SSc (45 cases with DUs and 45 cases without DUs) enrolled in this study. Patients with other rheumatologic diseases and diabetics patients were excluded from the study. Data which were collected from the patients, included, age, past medical history of cardiovascular disease and dyslipidemia, SSc disease duration, type of SSc (IcSSc or dcSSc), Raynaud's phenomenon (RP), RP duration and digital ulcerations (DUs), Body weight (BW), Height, Waist circumference (WC), Body mass index (BMI), Blood pressure (BP), serum levels of SCL-70 antibody and Anti $\beta 2$ microglobulin antibody. Then Doppler sonographies were performed. The outcome variables were the peak systolic velocity (PSV) and resistance index (RI) of ulnar, radial, popliteal, dorsalis pedis and tibia artery.

Results: The SSc patients with DUs have significantly lower PSV and higher RI in the ulnar (PSV:52. $1 \pm 7.9$ vs $55.7 \pm 4.1, p=0.006$ and $\mathrm{Rl}: 0.58 \pm 0.15$ vs $0.52 \pm 0.06$, $\mathrm{p}=0.003$ ), dorsalis pedis (PSV: $33.9 \pm 1.9$ vs $34.5 \pm 0.4, \mathrm{p}=0.027$ and $\mathrm{RI}: 0.54 \pm 0.11$ vs $0.50 \pm 0.04, p=0.045$ ) and tibial artery (PSV: $32.6 \pm 3.1$ vs $34.4 \pm 0.9, p<0.001$ and RI: $0.62 \pm 0.16$ vs $0.51 \pm 0.07, p<0.001$ ) in comparison to $S S c$ patients without DUs. PSV and RI of ulnar artery were significantly correlated with age $(\mathrm{p}=0.012$ and $p=0.019)$, disease duration ( $p=0.001$ and $p=0.001$ ) and Raynaud's phenomenon (RP) duration $(p=0.048$ and $p=0.028)$. PSV and $R I$ of tibial artery had significant correlation with age $(p=0.038$ and $p=0.009)$, systolic blood pressure $(p=0.022$ and $p=0.037)$ and diastolic blood pressure $(p=0.015$ and $p=0.010)$.

Conclusions: We concluded that digital ulceration in patients with SSc might be frequently related to the macrovascular dysfunction in below the elbow and knee. References:

[1] Walmsley D, Goodfield MJ. Evidence for an abnormal peripherally mediated vascular response to temperature in Raynaud's phenomenon. $\mathrm{Br} \mathrm{J}$ Rheumatol 1990;29:181-4.

[2] Chung L, Fiorentino D. Digital ulcers in patients with systemic sclerosis. Autoimmun Rev 2006;5:125-28.

[3] Ho M, Veale D, Eastmond C, Nuki G, Belch J. Macrovascular disease and systemic sclerosis. Ann Rheum Dis 2000;59:39-43.

[4] Veale DJ, Collidge TA, Belch JJ. Increased prevalence of symptomatic macrovascular disease in systemic sclerosis. Ann Rheum Dis 1995;54:853-5.

Disclosure of Interest: None declared

DOI: 10.1136/annrheumdis-2017-eular.1041

\section{AB0629 FROM LOCALIZED SCLERODERMA TO SYSTEMIC SCLEROSIS: A POSSIBLE EVOLUTION}

E. Cocchiara, C. Esposito, V. Raimondo, A. Spinella, F. Lumetti, M. Colaci, D. Giuggioli, C. Ferri on behalf of Rheumatology Unit, University of Modena and Reggio Emilia, Modena, Italy. Ospedale Policlinico di Modena, Modena, Italy

Background: Systemic Sclerosis (SSc) is a connective tissue disease characterized by skin fibrosis and visceral organ involvement. Localized Scleroderma (LoS), also known as morphea, is a fibrosing condition limited to the skin, subcutaneous tissue, underlying bone, and rarely central nervous system if present on face and head. SSc and LoS may share some aspects, such as histopathological findings, presence of autoantibodies and systemic symptoms, especially Raynaud phenomenon (RP). In this perspective they may represent two ends of a spectrum of disease.

Objectives: The aim of our study is to evaluate the evolution from LoS to SSc in our case series of SSc patients.

Methods: We retrospectively investigated 330 patients fulfilling the SScACR/EULAR criteria referred to our University-based Rheumatology Unit. The occurrence of LoS preceding the SSc diagnosis was evaluated for each patient on the basis of medical records; clinical, laboratory, and instrumental features were analyzed, from the presenting symptoms at the disease onset to the first visit and during the follow-up, with particular attention to very early cutaneous manifestations.

Results: Five SSc patients (1.5\% of our SSc series) had a clinical history of LoS prior to SSc diagnosis. All were women with mean age at time of LoS onset of $39 \pm 16.1 \mathrm{SD}$ years and time interval between LoS and SSc diagnosis of $19.2 \pm 16$ SD months. Skin biopsy was performed in $4 / 5$ patients showing nonspecific inflammatory infiltrate, collagen fiber deposition and dermis sclerosis. In all patients RP was the first extra-dermal symptom, preceding LoS in $2 / 5$ patients. Cutaneous involvement was represented by patches of skin sclerosis localized in limbs, trunk and face; while scleroderma was classified as cutaneous limited SSc in 4/5 patients and sine scleroderma in one. Following the SSc onset 2/5 patients had a history of digital ulcers, 1/5 esophagopathy, 1/5 interstitial lung disease; while capillaroscopy evidenced a SSc pattern in $4 / 5$ patients. ANA were detected in all patients with anti-ENA positivity in 3/5 (1 ACA, 1 anti-Scl70, 1 anti-U1RNP); the presence of autoantibodies was observed in $3 / 5$ individuals before SSc onset. None referred exposure to toxics or cigarettes smoke, while autoimmune thyropathy was the most frequent comorbidity. No local treatments had been employed for LoS but only low dosage of systemic steroids.

Conclusions: LoS and SSc are two distinct clinical entities that may share some clinical features; however, LoS is characterized by the absence of sclerodactyly, RP, digital ulcers, and typical SSc capillaroscopic changes; while possible internal organs involvement is much less frequently observed and the transition to SSC is exceptional and reported in only pediatric population. At our knowledge, this is the first observation of well-documented evolution from LoS to SSc in adult population as shown by updated review of the literature. The presence of RP and ANA positivity observed before the SSc onset can be considered as red flags of LoS evolution towards SSc, as reported in literature in pediatric population. SSc following LoS seems to be characterized by higher prevalence of vasculopatic symptoms compared to fibrotic complications. Finally, a careful clinical and laboratory monitoring of patients with LoS is recommendable to early identify the possible evolution to overt SSc.

Disclosure of Interest: None declared

DOI: 10.1136/annrheumdis-2017-eular.5120

\section{AB0630 CLINICAL SPECTRUM TIME COURSE COMPARISON BETWEEN PL-7, PL-12 AND EJ POSITIVE ANTISYNTHETASE SYNDROME}

E. Trallero Araguas ${ }^{1}$, A. Selva O'Callaghan ${ }^{1}$, C.A. Scire ${ }^{2}$, V. Codullo ${ }^{3}$, S. Castaneda ${ }^{4}$, N. Ortego Centeno ${ }^{5}$, J.A. Pereira da Silva ${ }^{6}$, C. Fiehn ${ }^{7}$, A. Schwarting ${ }^{8}$, A. Manfredi ${ }^{9}$, G. Emmi ${ }^{10}$, S. Barsotti ${ }^{11}$, O. Molberg $^{12}$,

A. Doria ${ }^{13}$, S. Parisi ${ }^{14}$, S. Scarpato ${ }^{15}$, N. Pipitone ${ }^{16}$, M. Piga ${ }^{17}$, I. Cavazzana ${ }^{18}$, F.J. Lopez-Longo ${ }^{19}$, J. Bachiller-Corral ${ }^{20}$, M. Benucci $^{21}$, J. Rojas-Serrano ${ }^{22}$, K. Triantafyllias ${ }^{23}$, N. Perez-Gomez ${ }^{24}$, G. Cagnotto ${ }^{25}$, F. Maurier ${ }^{26}$,

L. Quartuccio ${ }^{27}$, E. Bartoloni Bocci ${ }^{28}$, P. Tomietto ${ }^{29}$, R. Caporali ${ }^{3}$, F. lannone ${ }^{30}$, M.A. Gonzalez-Gay ${ }^{31}$, L. Cavagna ${ }^{3}$ on behalf of AENEAS collaborative group. ${ }^{1}$ Internal Medicine, Vall d'Hebron General Hospital, Barcelona, Spain;

${ }^{2}$ Reumatology, UOC Reumatologia, Azienda Ospedaliero Universitaria S. Anna, Ferrara; ${ }^{3}$ Rheumatology, University and Irccs Foundation Policlinico S. Matteo, Pavia, Italy; ${ }^{4}$ Rheumatology, Hospital Universitario de la Princesa, IIS-IP, Madrid; ${ }^{5}$ Systemic Autoimmune Diseases Unit, Hospital Clínico San Cecilio, Granada, Spain; ${ }^{6}$ Rheumatology, Centro Hospitalar e Universitário, Coimbra, Portugal; ${ }^{7}$ Rheumatology, ACURA Centre, Baden Baden; ${ }^{8}$ Rheumatology, University Hospital Johannes-Gutenberg, Mainz, Germany; ${ }^{9}$ Rheumatology, Azienda Ospedaliero-Universitaria Policlinico, Modena; ${ }^{10}$ Experimental and Clinical Medicine, University, Florence; ${ }^{11}$ Rheumatology, AOU Pisana, Pisa, Italy;

${ }^{12}$ Rheumatology, University Hospital, Oslo, Norway; ${ }^{13}$ Rheumatology, University, Padua: ${ }^{14}$ Rheumatology, Città della salute e della scienza, Torino;

${ }^{15}$ Rheumatology, ASL Salerno, Scafati; ${ }^{16}$ Rheumatology, S.Maria Hospital -IRCCS, Reggio Emilia; ${ }^{17}$ Rheumatology, University, Cagliari; ${ }^{18}$ Rheumatology, University and AO Spedali Civili, Brescia; ${ }^{19}$ Rheumatology, Hospital General Universitario Gregorio Marañón, Madrid, Italy; ${ }^{20}$ Rheumatology, University Hospital Ramón y Cajal, Madrid, Spain; ${ }^{21}$ Rheumatology, Azienda Sanitaria di Firenze, S. Giovanni di Dio Hospital, Firenze, Italy; ${ }^{22}$ Rheumatology, Instituto Nacional de Enfermedades Respiratorias, Ismael Cosío Villegas, Mexico city, Mexico; ${ }^{23}$ Rheumatology, ACURA Centre, Bad Kreuznach, Germany;

${ }^{24}$ Rheumatology, Hospital Clinico Universitario, Santiago de Compostela, Spain;

${ }^{25}$ Rheumatology, Skane University Hospital, Lund, Sweden; ${ }^{26}$ Medicine, HPMetz, Metz, France; ${ }^{27}$ Rheumatology, Santa Maria della Misericordia Hospital, Udine;

${ }^{28}$ Rheumatology, University, Perugia; ${ }^{29}$ Rheumatology, Cattnara Hospital, Trieste; ${ }^{30}$ Rheumatology, University, Bari, Italy; ${ }^{31}$ Rheumatology, Hospital Universitario Marqués de Valdecilla, IDIVAL, University of Cantabria, Santander, Spain

Background: Arthritis, myositis and Interstital lung disease (ILD) represent the classic clinical triad of antisynthetase syndrome (ASSD). In anti Jo-1 positive patients, these findings may appear also during the follow-up. Even if a similar cumulative trend has been showed also in non anti Jo-1 positive ASSD, a head to head comparison of clinical spectrum time course in these patients is still lacking Objectives: To assess the clinical spectrum time course in non anti Jo-1 positive ASSD, according to different underlying non anti Jo-1 specificities

Methods: Clinical, laboratory and instrumental data collection of anti PL-7, PL-12, and EJ positive patients from an international database of ASSD

Results: We identified 63 (42\%) anti PL-7, 66 (44\%) anti PL-12 and 20 (14\%) anti EJ positive patients, reporting their characteristics in table 1 (disease onset) and 2 (last follow-up). At disease onset, no substantial differences were observed. At the end of follow-up, we observed some differences between anti PL-12 and both anti PL-7 and anti-EJ positive patients. In particular, anti PL-12 positive patients presented less frequently ex-novo triad findings and had a reduced prevalence of 